\title{
Plasticity of fibroblasts demonstrated by tissue-specific and function-related proteome profiling
}

Astrid Slany ${ }^{1}$, Anastasia Meshcheryakova², Agnes Beer ${ }^{2}$, Hendrik Jan Ankersmit ${ }^{4}$, Verena Paulitschke ${ }^{3}$ and Christopher Gerner ${ }^{1,2^{*}}$

\begin{abstract}
Background: Fibroblasts are mesenchymal stromal cells which occur in all tissue types. While their main function is related to ECM production and physical support, they are also important players in wound healing, and have further been recognized to be able to modulate inflammatory processes and support tumor growth. Fibroblasts can display distinct phenotypes, depending on their tissue origin, as well as on their functional state.

Results: In order to contribute to the proteomic characterization of fibroblasts, we have isolated primary human fibroblasts from human skin, lung and bone marrow and generated proteome profiles of these cells by LC-MS/MS. Comparative proteome profiling revealed characteristic differences therein, which seemed to be related to the cell's tissue origin. Furthermore, the cells were treated in vitro with the pro-inflammatory cytokine IL-1 beta. While all fibroblasts induced the secretion of Interleukins IL-6 and IL-8 and the chemokine GRO-alpha, other inflammation-related proteins were up-regulated in an apparently tissue-dependent manner. Investigating fibroblasts from tumorous tissues of skin, lung and bone marrow with respect to such inflammation-related proteins revealed hardly any conformity but rather individual and tumor type-related variations. However, apparent up-regulation of IGF-II, PAI-1 and PLOD2 was observed in melanoma-, lung adenocarcinoma- and multiple myeloma-associated fibroblasts, as well as in hepatocellular carcinoma-associated fibroblasts.

Conclusions: Inflammation-related proteome alterations of primary human fibroblasts were determined by the analysis of IL-1 beta treated cells. Tumor-associated fibroblasts from different tissue types hardly showed signs of acute inflammation but displayed characteristic functional aberrations potentially related to chronic inflammation. The present data suggest that the state of the tumor microenvironment is relevant for tumor progression and targeted treatment of tumor-associated fibroblasts may support anti-cancer strategies.
\end{abstract}

Keywords: Fibroblasts, Inflammatory activation, Tumor-stroma interactions, Primary human cells, Proteome profiling

\section{Background}

Fibroblasts have long been considered as rather simple structural cells contributing to the extracellular matrix $(E C M)$ in connective tissues and responsible for tissue repair during wound healing. In the last years, however, these cells have been recognized to play an important role

\footnotetext{
*Correspondence: christopher.gerner@univie.ac.at

${ }^{1}$ Faculty of Chemistry, Department of Analytical Chemistry, University of Vienna, Vienna, Austria

${ }^{2}$ Department of Medicine I, Institute of Cancer Research, Medical University of Vienna, Austria; Christian Doppler Laboratory for the Diagnosis and Regeneration of Cardiac and Thoracic Diseases, Medical University Vienna, Vienna, Austria

Full list of author information is available at the end of the article
}

in other processes as well, being able to exert a significant influence on all kind of cells in their environment. So, it appears now evident that fibroblasts are decisive players in inflammatory processes, modulating the activities of leukocytes by secreting specific signaling molecules at sites of inflammation [1]. Importantly, fibroblasts seem to be responsible for the termination of immune responses, or alternatively, for the switch from acute to chronic inflammation [2,3]. During acute inflammation, immune cells are recruited and expanded in the damaged tissue. Under normal physiological conditions, control mechanisms prevent from over-stimulating these cells. Hereby 
fibroblasts play an important role by releasing immuneregulatory signals which promote the removal of dead or redundant immune cells. Chronic inflammation may occur when these clearance mechanisms fail. This may happen when fibroblasts either inhibit apoptotic processes by producing survival cytokines, or retain the immune cells by the release of specific chemokines [2]. Chronic inflammation processes are also involved in diseases such as fibrosis and cancer. Regulatory effects of fibroblasts on the development and progression of fibrosis and cancer have been demonstrated [4-8]. Reorganization of the ECM by fibroblasts plays thereby an important role. In tumors, additionally, interactions between fibroblasts and tumor cells seem to be essential for tumor growth and progression. These interactions are mediated through soluble signaling factors such as growth factors, cytokines, chemokines and lipid products, or by direct communication of the cells through integrins [9] ECM-degrading enzymes such as matrix metalloproteinases (MMPs), produced by fibroblasts, contribute to the degradation and remodeling of the tumor environment, thereby supporting tumor progression, including angiogenesis, invasiveness and metastasis [10].

It appears nowadays clear that fibroblasts are not only involved in structural concerns, but are also important players in patho-physiological processes. However, a lot of questions remain unanswered. So, the characterization of different fibroblast sub-types, including their expression profiles in inactivated as well as in activated and disease-related cell states, is still not clearly assessed. Up until now, no selective markers have been established, and cells with spindle-like morphology, which in-vitro adhere on plastic ware, and furthermore have nonlymphoid, non-endothelial and non-epithelial phenotypes, are generally considered as fibroblasts [1]. Some attempts have therefore been made to better characterize these cells $[4,11,12]$. However, the results of these investigations are raising new questions. In effect, it seems that more different sub-types of fibroblasts exist than expected [13]. Some researchers even claim to define new cell types and point out the fact that fibroblasts from different anatomic sites display differences which are comparable to those observed among different lineages of leukocytes [14]. The possibility cannot be excluded that multiple sub-types are co-localized at a single location. Furthermore, the origin of fibroblasts at a specific site cannot be accurately determined. They may arise from the primary mesenchyme, or alternatively from BM derived precursor cells, or from local epithelial-mesenchymal transition (EMT) [6,15]. Knowledge about specific marker molecules, which could distinguish between fibroblasts from different anatomic sites, as well as between fibroblasts at different functional or disease-related states, would massively contribute to the understanding of patho- physiological mechanisms. Furthermore, in cancer, mortality is often related to distant metastases which rather occur as late steps of disease progression. Early cancer markers are therefore urgently needed to allow prompt treatments before aggressive forms of cancer can develop. Such early disease markers could be found in the altered stromal microenvironment of the tumor, whose main contributors are fibroblasts. Characterization of exact phenotypes of normal as well as of activated and diseaserelated fibroblasts would be of particular importance in this context. As different functionalities are accompanied by the synthesis or up-regulation of specific proteins, they may be revealed by expression or proteome profiling experiments.

The aim of this study was to contribute to the proteomic characterization of fibroblasts from different anatomic sites and at different functional and disease-related cell states. Proteome profiles of normal human lung fibroblasts were generated and compared to those previously obtained from primary skin and BM fibroblasts. The aim was to work out characteristic proteome signatures for these cells. Furthermore, the same cells were treated in an inflammatory way using IL-1 $\beta$, in order to find out function-specific proteome alterations. Finally, in a last step we aimed to determine the functional states of tumor-associated fibroblasts from analogous tissues. On the one hand the proteome profile of lung cancerassociated fibroblasts was analyzed. On the other hand, previously published proteomic data obtained from the analysis of fibroblasts related to melanoma and thus representing cancer-associated skin fibroblasts were used, as well as fibroblasts obtained from patients with multiple myeloma, a plasma cell tumor of the BM $[16,17]$. Furthermore, results obtained from our previous investigations concerning HCC-associated fibroblasts [18] were included in the present study for comparative analyses. The aim was to answer the questions if tumor-associated fibroblasts manifest an inflammatory activated cell state, and if fibroblasts related to different cancers may reveal similarities regarding their proteome profiles.

\section{Results and discussion}

\section{Experimental strategy}

An important aim of this study was to determine marker proteins which would allow distinguishing different phenotypes of fibroblasts. The first objective was to find out if it was possible to discern proteome signatures characteristic for fibroblasts from different tissue origins. Comparative proteome profiling was used to clarify this question. Fibroblasts isolated from different tissues, namely from skin, lung and BM, analyzed by LC-MS/MS based proteome profiling, were compared. Moreover, these cells were analyzed at different functional states, i.e. in non-activated, an inflammatory activated and tumor- 
associated states. This allowed us to characterize functionrelated proteome signatures as well. Beside characteristic features of tumor-associated fibroblasts, the inflammatory status of these cells was of special interest.

\section{Cell culture and characterization of primary cells, as well as treatment of cells with IL-1 $\beta$}

Primary human lung fibroblasts isolated both, from tumor, as well as from non-tumor tissue areas were cultivated for three passages in order to get enough cells for further analyses. Compatible with our observations, Chang et al. have demonstrated that fibroblasts maintain a stable phenotype for several passages during cell culture conditions [14]. The cells were then characterized by FACS analysis (Additional file 1: Figure S1), which revealed that they were highly positive for CD90, but negative for leukocyte, endothelial cell and hematopoietic stem cell markers CD45, CD31 and CD34, proving the purity of the cell preparations. Furthermore, the cells showed typical fibroblast's characteristics, such as adherent growth on culture flasks, as well as a spindle-like morphology. $41.3 \%$ and $62.1 \%$ of the cells from non-cancerous and cancerassociated areas, respectively, were positive for CD54 (ICAM-1), which indicated inflammatory activation [19] in both kinds of cells, even though more cancer-associated fibroblasts were activated than non-cancerous ones. Furthermore, $34 \%$ of cancer-associated fibroblasts were positive for alpha-smooth muscle actin ( $\alpha$-SMA), corresponding to a myofibroblast phenotype characteristic for cancerassociated fibroblasts [20]. However, $21.5 \%$ of the fibroblasts from non-cancerous areas showed $\alpha$-SMA-positive staining as well, indicating a slight amount of $\alpha$-SMA-positive cells in this cell population. For comparison, we also included cells acquired by purchase, namely NHLF, in our investigations as reference cell system for normal lung fibroblasts. NHLF were used in their non-activated cell state, or alternatively treated for 24 hours with IL- $1 \beta$ in order to generate fibroblasts in an inflammatory activated cell state. Skin- and BM-derived fibroblasts, raised as previously described $[17,21]$, were treated with IL-1 $\beta$ in the same way.

\section{Generation of proteome profiles of primary human fibroblasts at different functional cell states}

In a first step, proteome profiles of sub-cellular fractions (cytoplasm, secretome and nuclear extract) of NHLF and primary lung fibroblasts were generated. Data of the sub-cellular fractions were afterwards combined in order to obtain comprehensive proteome profiles for each cell type, which resulted in a total of 1577 and 1545 proteins for NHLF and primary lung fibroblasts, respectively (Additional file 2: Table $\mathrm{S} 1$ and Additional file 3: Table S2). For comparative analyses, which was supported by our specially designed database, the GPDE (see Methods), the proteome profile of skin fibroblasts, comprising 1496 proteins (Additional file 4: Table S3), as well as that of BM-derived fibroblasts, including 1569 proteins (Additional file 5: Table S4) were further used. Parts of these proteome profiles have been published previously $[17,21]$. However, the present protein lists may differ slightly as for comparative analysis we now also included one peptide-identifications when the corresponding protein was identified in any other of the here investigated samples with two or more peptides.

Proteome profiles of the inflammatory activated cells were generated similarly, identifying 1608 proteins in activated NHLF (Additional file 6: Table S5), 1428 proteins in activated skin fibroblasts (Additional file 7: Table S6), and 1347 proteins in activated BM fibroblasts (Additional file 8: Table S7). Finally, for the consideration of tumorassociated fibroblasts we again made use of previously published proteome profiles obtained from the analysis of melanoma-associated skin fibroblasts [16], multiple myeloma-associated BM fibroblasts [17] and HCCassociated fibroblasts [18]. The respective proteome profiles are summarized in Additional file 9: Table S9, Additional file 10: Table S10, Additional file 11: Table S11. In addition, proteome profiles of lung cancer-associated fibroblast were analyzed, which resulted in a total of 1454 identified proteins (Additional file 12: Table S8).

\section{Primary human fibroblasts from different tissue types display differences in their proteome profiles}

Stromal fibroblasts have several common biological tasks irrespective of the tissue origin or functional state of the cells. Such tasks include generation and maintenance of the ECM and provision of physical support and elasticity, and may be related to specific protein synthesis. A similarity in the proteome profiles regarding proteins related to such functions should therefore be observed in fibroblasts, irrespective of their tissue origin. Indeed, several ECM-related proteins such as fibrillin-1 and different subtypes of collagens were identified by us in all fibroblasts isolated from skin, lung and BM, irrespective of the functional states of the cells (Figure 1). Furthermore, in all kinds of fibroblasts we further detected ECM modulating enzymes such as procollagen $\mathrm{C}$-endopeptidase enhancer 1, which contributes to the cross-linking of ECM proteins [22], as well as MMP-2 and c-type mannose receptor 2, which are both involved in collagen degradation, as described previously [17]. CD90, procollagen $\mathrm{C}$-endopeptidase enhancer 1 , and c-type mannose receptor 2 were listed in the proteome signature specific for fibroblasts, published recently [21].

Beside such common features characteristic for all types of fibroblasts, we expected some functional specialization of the cells, related to the tissue origin. Therefore, the data obtained from skin, lung and BM fibroblasts, comprising all data from control, inflammatory activated and tumor- 
Skin fibroblasts:

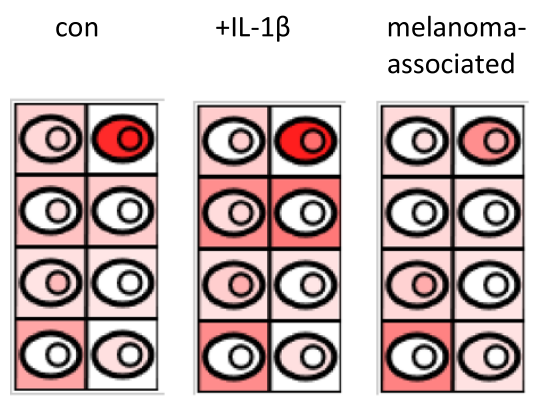

Lung fibroblasts:

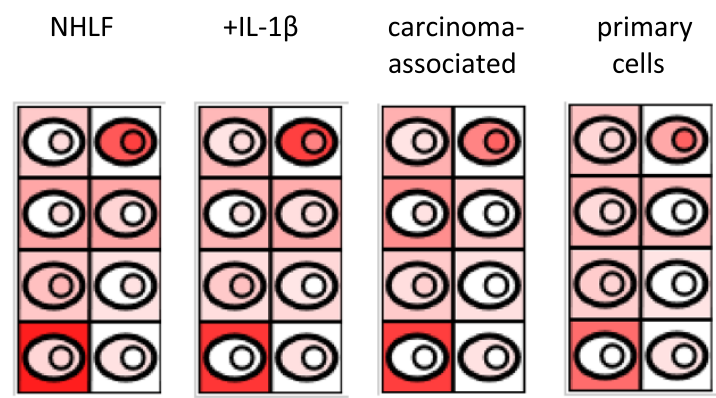

BM fibroblasts:

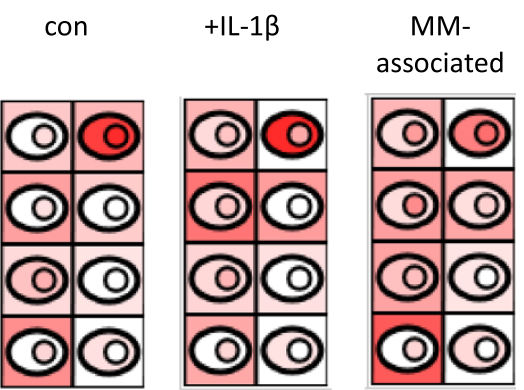

Liver fibroblasts:

HCC-

associated

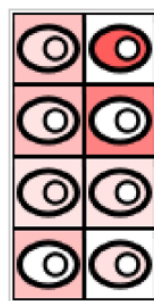

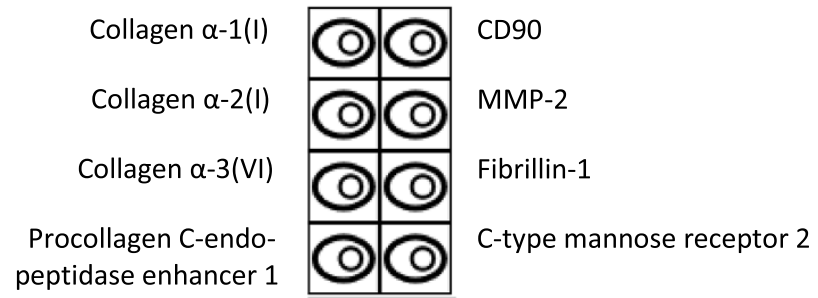

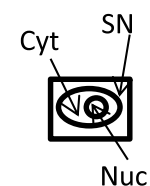

Figure 1 Proteins which are found in all kinds of fibroblasts. Based on the proteome profiles of skin, bone marrow (BM), lung and liver fibroblasts in different functional states, a semi-quantitative assessment of selected proteins in these cells was performed using the corresponding emPAl values (see Methods). The emPAI values for all sub-cellular fractions are visualized by colored cell symbols, increased color intensities corresponding to increased protein abundances. Con, non-activated control cells; $+\mathrm{IL}-1 \beta, \mathrm{IL}-1 \beta$ treated cells; Cyt, cytoplasmic fraction; SN, cell supernatant; Nuc, nuclear fraction; NHLF, normal human lung fibroblasts; MM, derived from multiple myeloma patients; HCC, derived from hepatocellular carcinoma patients.

associated fibroblasts were searched for proteome signatures potentially characteristic for the fibroblast's tissue origin. Comparative proteome profiling searching for proteins detected exclusively in fibroblasts of one tissue origin suggested the existence of tissue-specific signatures. Taking the close relation of cell functions into account, proteins detected in fibroblasts from two kinds of tissue origins but absent from the third tissue were considered as well. All selected proteins were verified regarding their biological functionalities and regarding known literature about tissue specificity. The finally compiled signatures, each potentially characteristic for fibroblasts of one tissue type are listed in Figure 2, with corresponding emPAI values listed in Additional file 13: Table S12. We thus suggest that the tissue of origin of fibroblasts may be determined making use of such signatures; the practical applicability thereof needs to be established in the future.

The presently suggested signature characteristic for skin fibroblasts contained dermatopontin, slit homolog 3 protein, PDGFR-beta, LIM domain only protein 7 , seprase, secreted frizzled-related protein 1, and MMP-1 (Figure 2, rose colored). Dermatopontin is an abundant dermal extracellular matrix protein, contributing to the communication of dermal fibroblasts with their environment [23]. Slit homolog 3 protein plays an important role as general cell migration guidance molecule, supporting epidermal cell migration [14]. Remarkably, Denk et al. have observed that this protein may be hindering the migration of 


\begin{tabular}{|c|c|c|c|c|c|c|c|c|c|c|}
\hline AccNr & Protein name & $\begin{array}{l}\text { Skin } \\
\text { fibs }\end{array}$ & $\begin{array}{l}\text { Skin fibs } \\
+1 \mathrm{L1b}\end{array}$ & $\begin{array}{l}\text { Melanoma- } \\
\text { assoc. fibs }\end{array}$ & NHLF & $\begin{array}{l}\text { NHLF + } \\
\text { IL1b }\end{array}$ & $\begin{array}{l}\text { Lung } \\
\text { Cancer- } \\
\text { assoc. fibs }\end{array}$ & BM fibs & $\begin{array}{l}\text { BM fibs + } \\
\text { L1b }\end{array}$ & $\begin{array}{l}\text { MM- } \\
\text { assoc. } \\
\text { fibs }\end{array}$ \\
\hline 0,07507 & Dermatopontin & ++ & ++ & + & - & - & - & - & - & - \\
\hline 075094 & $\begin{array}{l}\text { Slit homolog } 3 \\
\text { protein }\end{array}$ & ++ & ++ & - & - & - & - & - & - & - \\
\hline $\begin{array}{l}\text { P09619 } \\
\end{array}$ & PDGFR-beta & ++ & + & + & - & - & - & - & - & +++ \\
\hline Q8WWI1 & $\begin{array}{l}\text { LIM domain only } \\
\text { protein } 7\end{array}$ & +++ & ++ & - & - & - & - & ++ & +++ & ++++ \\
\hline Q12884 & Seprase/ FAP & ++ & - & - & - & - & - & ++ & ++ & ++ \\
\hline P19320 & VCAM-1/CD106 & - & - & - & - & - & - & ++ & ++ & ++ \\
\hline Q992954 & Proteoglycan 4 & - & - & - & - & - & - & ++ & + & + \\
\hline Q12929 & $\begin{array}{l}\text { EGF receptor } \\
\text { kinase substrate } 8\end{array}$ & - & - & - & - & - & - & ++ & - & ++ \\
\hline P36222 & $\begin{array}{l}\text { Chitinase 3-like } \\
\text { protein 1 }\end{array}$ & - & - & - & - & - & ++ & ++++ & +++ & - \\
\hline Q8WWM9 & Cytoglobin & - & - & - & ++ & ++ & + & ++ & ++ & +++ \\
\hline P17900 & $\begin{array}{l}\text { Ganglioside GM2 } \\
\text { activator }\end{array}$ & - & - & - & ++ & ++ & - & ++ & - & ++ \\
\hline P29279 & CTGF & - & - & - & + & + & + & + & + & ++ \\
\hline 075787 & Renin receptor & - & - & - & ++ & ++ & ++ & - & ++ & - \\
\hline Q9BUD6 & Spondin-2 & - & - & - & +++ & ++ & +++ & - & - & - \\
\hline 000468 & Agrin & - & - & - & ++ & ++ & - & - & - & - \\
\hline Q8N474 & $\begin{array}{l}\text { Secreted frizzled- } \\
\text { related protein } 1\end{array}$ & - & - & + & ++ & - & ++ & - & - & - \\
\hline P03956 & $\begin{array}{l}\text { MMP-1/ fibroblast } \\
\text { collagenase }\end{array}$ & +++ & +++ & ++ & +++ & ++++ & & - & - & - \\
\hline
\end{tabular}

Figure 2 Protein signatures characteristic for fibroblasts of different tissue origins. The total number of distinct peptides identified per protein when considering all three sub-cellular fractions was used as a semi-quantitative measure for protein abundances in the different fibroblasts. AccNr, Swiss-Prot accession number; -, protein was not detected; +, protein was detected with one peptide; ++, protein was detected with 2 to 5 peptides; +++ , protein was detected with $\geq 6$ peptides; ++++ , protein was detected with $\geq 15$ peptides.

malignant melanoma cells [24]. Compatible with this notion, an apparent down-regulation of this protein in melanoma-associated skin fibroblasts was observed. An intriguing idea might be that melanoma cells may influence neighboring fibroblasts in terms of down-regulation of this protein which would otherwise restrict their motility. PDGFR-beta was detected by us in all kinds of skin fibroblasts, as well as in in multiple myeloma-associated BM fibroblasts, which we have discussed previously [17]. The observation that BM fibroblasts up-regulate this protein only when they are in a proliferation state was further supported by others [25]. PDGFR-beta has also been described to occur in lung fibroblasts [26], however, the protein was not detected in such cells analyzed by us. MMP-1 was detected by us in all kinds of skin fibroblasts and in control as well as in inflammatory activated lung fibroblasts, but not in BM-derived cells. Halfon et al. have described MMP-1 as one of the proteins which may be a suitable marker for skin and lung fibroblasts allowing distinguishing them from BM-derived mesenchymal stromal cells [27], which is compatible with the present interpretation. Adenocarcinoma-related cells apparently express lower amounts of MMP-1 than other lung cancers [28], which may explain why MMP-1 was not detected by us in cancer-associated lung fibroblasts.

The presently suggested signature characteristic for BM-derived fibroblasts included PDGFR-beta, LIM domain only protein 7 , seprase, V-CAM-1, proteoglycan 4 , EGF receptor kinase substrate 8, chitinase 3-like protein 1, cytoglobin, ganglioside GM2 activator, as well as CTGF and renin receptor (Figure 2, green colored). VCAM-1 is known since long to be produced by BM fibroblasts [29].
Proteoglycan 4 has been described to be predominantly synthesized by BM stromal cells, regulating immune cells and parathyroid hormone actions on BM hematopoietic progenitor cells [30,31]. Seprase (also called fibroblast activation protein alpha) has been proposed as candidate marker protein for BM mesenchymal stromal cells by Bae et al. [32]. This protein was detected by us in all kinds of BM fibroblasts, but also in control skin fibroblasts. LIM domain only protein 7 was similarly detected in all kinds of BM fibroblasts, the up-regulation observed in inflammatory activated and tumor-associated BM fibroblasts correlating with that observed in breast cancer-associated stromal cells; the protein seems to be important for cancer cell migration [33]. One of the proteins detected in BM-fibroblasts and NHLF, but not in skin fibroblasts, cytoglobin, has similarly been described by Nakatani et al. to be present in several fibroblasts, but not in dermal ones [34]. Chitinase 3-like protein 1, previously described by us [17], was found to be downregulated in tumor-associated BM fibroblasts. In lung cancer-associated fibroblasts, on the other hand, it was found by us to be up-regulated relative to control cells, which was supported by observations of others [35].

Finally, the presently suggested proteome signature characteristic for lung-derived fibroblasts comprised chitinase 3-like protein 1, cytoglobin, ganglioside GM2 activator, CTGF, renin receptor, spondin-2, agrin, secreted frizzled-related protein 1, and MMP-1 (Figure 2, blue colored). Actually, spondin-2, together with secreted frizzledrelated protein 1 , is required for lung morphogenesis. These two proteins have antagonistic effects on the Wnt signaling pathway which is critical in the induction of cell 
differentiation necessary for the formation of organs such as the lung [36,37]. Agrin has also been described in the context of lung, apparently being up-regulated in the basement membranes of adult lungs, probably playing a role as mediator in pulmonary function [38]. Concerning renin receptor, this protein may be involved in the reninangiotensin system, which is regulating the blood pressure, but seems also to play a role in lung cell inflammation and proliferation $[39,40]$.

\section{Primary human fibroblasts from different tissue origins display differences in their inflammatory signature} In order to find out characteristics related to an inflammatory activated state of fibroblasts, proteome profiles of lung, skin and BM fibroblasts treated with IL-1 $\beta$ were generated (Additional file 6: Table S5, Additional file 7: Table S6 and Additional file 8: Table S7) and compared with those of the corresponding control cells (Additional file 2: Table S1, Additional file 4: Table S3 and Additional file 5: Table S4). The efficiency of inflammatory activation in response to stimulation with IL- $1 \beta$ was confirmed by the detection of induced proteins known to be upregulated during an inflammatory response. So, IL-6 and IL-8, classic inflammatory cytokines, were readily identified in all fibroblasts upon inflammatory activation, just as GRO-alpha (CXCL1) and CXCL6, both chemotactic for neutrophils (Table 1, and corresponding emPAI values in Additional file 14: Table S13; Figure 3). Thus, all these fibroblasts proved to be prepared to respond to an inflammatory stimulus and reacted promptly to the IL-1 $\beta$ treatment. Two other proteins, PLOD- 2 and palladin were likewise found to be up-regulated in all three kinds of inflammatory activated cells. PLOD-2 is necessary for cross-linking collagens. Up-regulation of the

Table 1 Proteins differentially regulated in different fibroblasts upon inflammatory activation

\begin{tabular}{|c|c|c|c|c|c|c|c|}
\hline \multirow{2}{*}{$\begin{array}{l}\text { AccNr } \\
\text { Supernatant: }\end{array}$} & \multirow[t]{2}{*}{ Protein name } & \multicolumn{2}{|c|}{ Skin fibroblasts } & \multicolumn{2}{|l|}{ NHLF } & \multicolumn{2}{|c|}{ BM fibroblasts } \\
\hline & & Control & IL-1 $\beta$-treated & Control & IL-1 $\beta$-treated & Control & IL-1 $\beta$-treated \\
\hline P05231 & $\mathrm{IL}-6$ & - & +++ & - & ++ & - & +++ \\
\hline P10145 & $\|-8$ & - & +++ & - & ++ & - & ++ \\
\hline P09341 & GRO-alpha/CXCl1 & - & ++ & - & +++ & - & ++ \\
\hline P80162 & CXCL6 & - & ++ & - & ++ & - & ++ \\
\hline P42830 & CXCL5 & - & ++ & - & ++ & - & - \\
\hline P08254 & MMP-3/Stromelysin-1 & - & +++ & - & +++ & - & - \\
\hline P07585 & Decorin & ++ & ++++ & +++ & ++++ & ++ & ++ \\
\hline P08253 & MMP-2 & ++ & ++++ & +++ & +++ & ++ & +++ \\
\hline P09603 & CSF-1 & - & ++ & ++ & ++ & - & ++ \\
\hline P98066 & TSG-6 & - & ++ & - & - & - & ++ \\
\hline P26022 & PTX3/ TSG-14 & +++ & +++ & - & ++ & +++ & ++++ \\
\hline P19875 & GRO-beta /CXCL2 & - & ++ & - & - & - & - \\
\hline P05121 & PAI-1 & +++ & +++ & ++ & +++ & +++ & +++ \\
\hline О00391 & Sulfhydryl oxidase 1 & $(+)$ & ++ & ++++ & +++ & - & ++ \\
\hline Q15063 & Periostin & $(+)$ & - & ++ & +++ & $(+)$ & ++ \\
\hline Q9Y240 & SCGF & ++ & - & $(+)$ & ++ & - & - \\
\hline \multicolumn{8}{|l|}{ Cytoplasm: } \\
\hline 000469 & PLOD-2 & $(+)$ & ++ & - & +++ & $(+)$ & +++ \\
\hline P05362 & ICAM-1 (CD54) & - & - & $(+)$ & + & - & ++ \\
\hline P05120 & PAI-2 & ++ & +++ & - & +++ & ++ & - \\
\hline \multicolumn{8}{|c|}{ Nuclear extract: } \\
\hline Q8WX93 & Palladin & ++ & +++ & ++ & +++ & ++ & +++ \\
\hline P51911 & Calponin-1 & +++ & +++ & ++ & +++ & ++ & +++ \\
\hline Q16666 & y-IFN-inducible protein 16 & ++ & ++ & ++ & ++ & ++ & +++ \\
\hline
\end{tabular}

The total number of distinct peptides identified per protein when considering all three sub-cellular fractions was used as a semi-quantitative measure for protein abundances in the different fibroblasts, which allowed determining up- or down-regulation of proteins upon inflammatory activation. Most of the regulated proteins were found in the secretomes, listed under "Supernatant".

AccNr, Swiss-Prot accession number; -, protein was not detected; +, protein was detected with one peptide; ++, protein was detected with 2 to 5 peptides; +++ , protein was detected with $\geq 6$ peptides; ++++ , protein was detected with $\geq 15$ peptides; $(+)$, protein identification was ambiguous, i.e. protein was not found robustly in all corresponding samples. 
protein was described to occur during inflammatory activation of skin and lung fibroblasts [41,42]. Furthermore, in several fibrotic diseases, which are characterized by chronic inflammation and by the deposition of ECM components such as collagenous fibers, PLOD-2 seems to be closely involved in disease progression. Wu et al. have demonstrated that in dermal fibrosis, PLOD-2 upregulation also leads to an overexpression of collagen alpha-1(I) which contributes to the development of this skin disease [43]. In the BM, PLOD-2 overexpression has been observed to account for the progression of primary myelofibrosis [44]. Concerning palladin, this protein has been described to be up-regulated in the leading edge of wounds [45]. Apparently, it seems to be involved in wound healing processes, accompanying TGF-beta-1 induced myofibroblast differentiation, and is possibly necessary for the generation of contractile forces in these cells and the transmission of these forces to the ECM [46].

In addition, apparent tissue-related differences in the responses to the inflammatory stimulus (Table 1) were also observed. Actually, some of the proteins were found to be up-regulated only in one type of fibroblasts. So, GRO-beta (CXCL2) was only detected in activated skin fibroblasts, PAI-1 in activated lung fibroblasts, and $\gamma$-IFN-inducible protein 16 in activated BM fibroblasts. This latter protein, even though also described to be involved in senescence of fibroblasts [47], is considered primarily to play an important role in inflammatory processes. Indeed, it seems to be a critical, predominantly nuclear sensor for detecting pathogenic DNA and inducing pro-inflammatory responses [48]. In endothelial cells, the protein has been described to be necessary for IFN- $\gamma$ triggered stimulation of pro-inflammatory genes through NF-kB, which apparently leads to the up-regulation of adhesion molecules such as ICAM-1, as well as chemokines and cytokines such as IL-8 and CXCL6 [49]. We found upregulation of this protein in IL- $1 \beta$ treated BM-derived fibroblasts, showing that fibroblast as well may be able to respond to an inflammatory stimulus through $\gamma$ IFN-inducible protein 16.

Other proteins were found by us to be up-regulated by IL- $1 \beta$ in two kinds of fibroblast, such as MMP-3 in activated skin and lung fibroblasts, MMP-2 in activated skin and BM fibroblasts and ICAM-1 in activated lung and BM fibroblasts. Furthermore, three proteins displayed oppositional modes of regulation. So, PAI-2 was found to be up-regulated in activated skin and lung fibroblasts, but down-regulated in activated BM fibroblasts; sulfhydryl oxidase 1 was apparently upregulated in activated skin and BM fibroblasts, but down-regulated in activated lung fibroblasts; and SCGF was up-regulated in activated lung fibroblasts, down-regulated in activated skin fibroblasts, and not detected by us in BM fibroblasts. The majority of these proteins can immediately be related to an inflammatory related mechanism, most of them being induced through IFN- $\gamma$ and NFKB [50-55]. Therefore, our observations may suggest tissue-type specific modulation of inflammation-related processes.

\section{A}
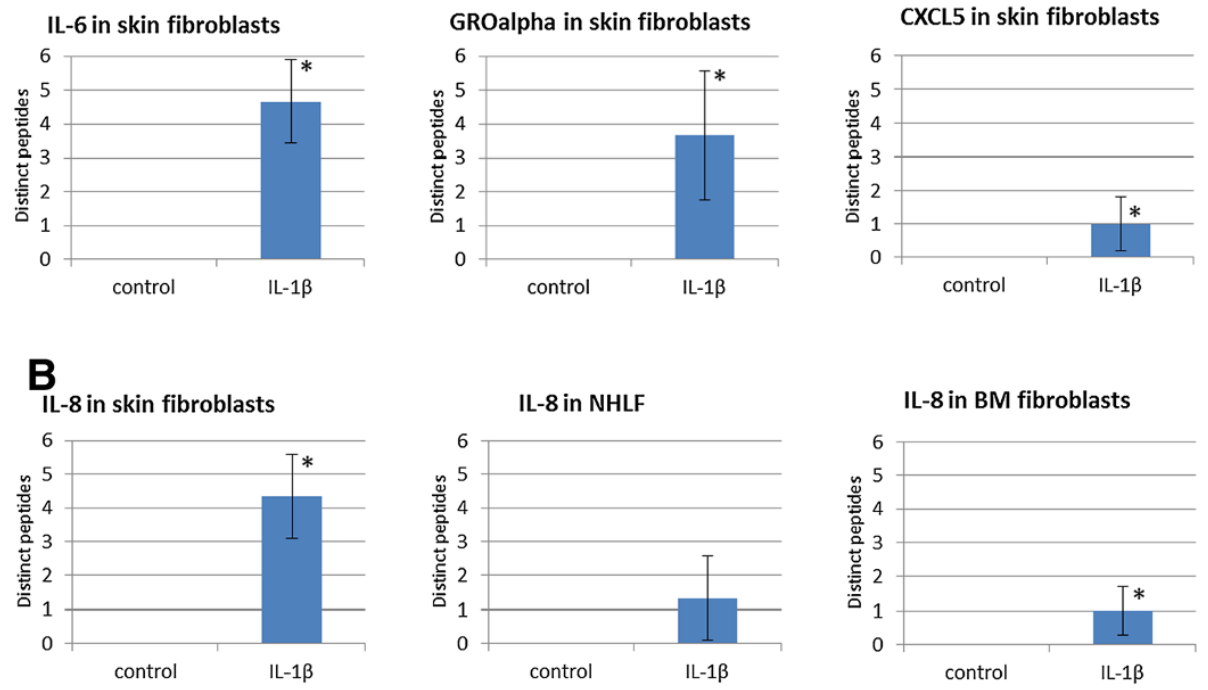

Figure 3 Up-regulation of selected proteins in the cell supernatant of fibroblasts upon IL-1 $\beta$ treatment. Successful inflammatory activation in fibroblasts upon IL-1 $\beta$ treatment was demonstrated by up-regulation of known inflammation-induced proteins. The mean number of distinct peptides identified per protein in the respective cell supernatants, determined from at least three independent experiments, was used. Significant up-regulation was determined using the Chi-squared test (*, $p<0.05)$. (A) Up-regulation of IL-6, GRO-alpha and CXCL5 in IL-1 $\beta$ treated skin fibroblasts. (B) Up-regulation of IL-8 in IL-1 $\beta$ treated skin, lung (NHLF) and BM fibroblasts. 
Characterization of tumor-associated fibroblasts _ PLOD-2 and PAI-1 as possible markers for chronic inflammation and IGF-II as potential marker for tumor-associated fibroblasts

As fibroblasts seem to be highly involved in chronic inflammatory processes, and as further chronic inflammation seems to play an important role for tumor development, we wanted to assess the inflammation status of tumorassociated fibroblasts. Tumor-associated fibroblasts related to skin, lung, BM, as well as liver, namely from melanoma, lung adenocarcinoma, multiple myeloma and HCC were analyzed. For HCC it is well-known that acute as well as chronic inflammatory processes play an important role in disease progression [56]. Actually, in HCC-associated fibroblasts we found up-regulation of the classical inflammation mediators, IL-6, IL-8 and GRO-alpha, which are proteins up-regulated in the early phase of inflammation. Those were not detected in any other tumor-associated fibroblasts, as demonstrated for IL-6 in Figure 4. However, in all kinds of tumor-associated fibroblasts we observed up-regulation of PAI-1 and PLOD-2 (Figure 4). PLOD-2 has been described to be up-regulated in several fibrotic

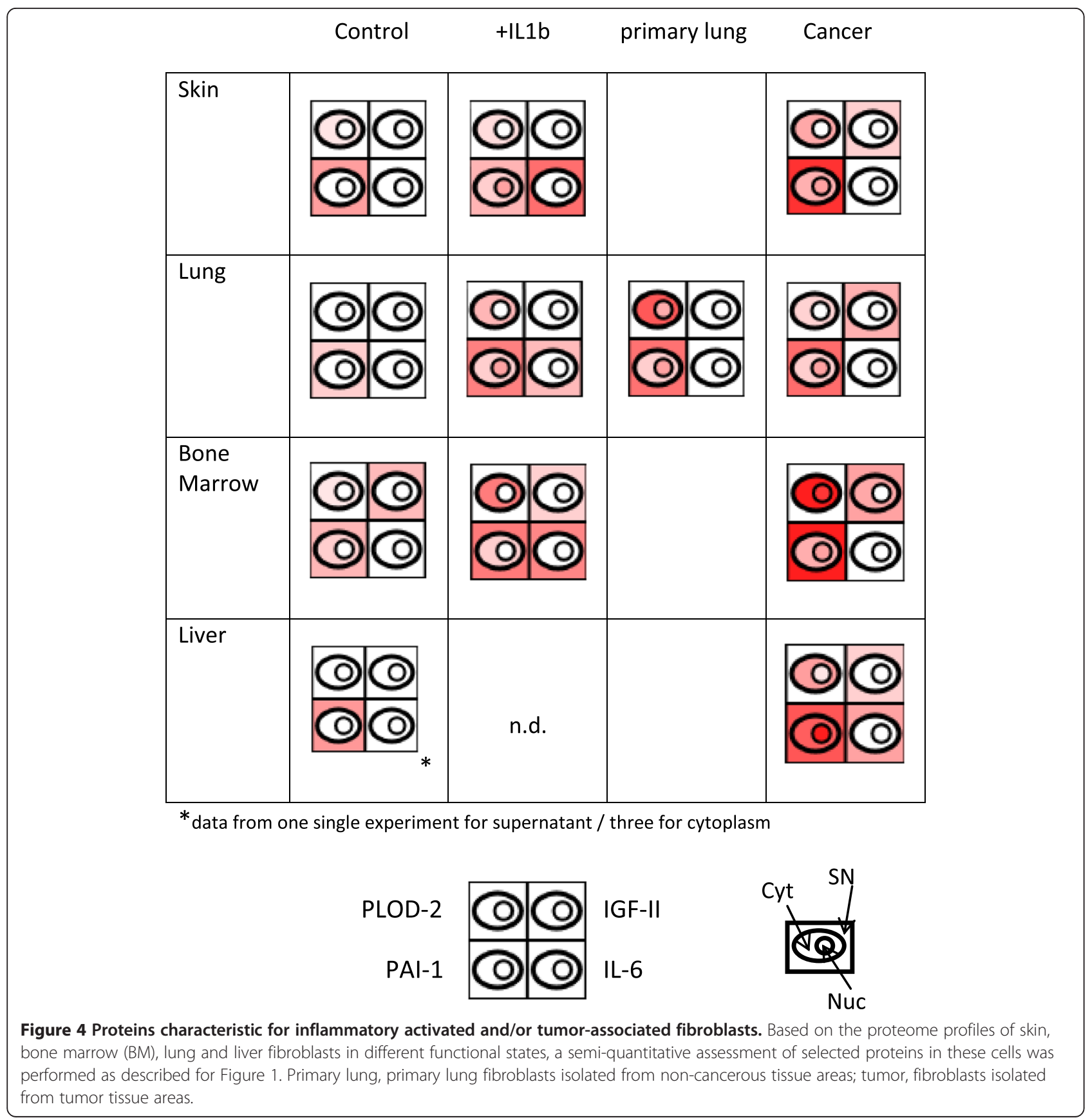


diseases characterized by chronic inflammation, as mentioned above. Interestingly, PLOD-2 has recently been proposed as a marker protein for different cancer-associated fibroblasts by a method based on RNA profiling data [57]. Moreover, PLOD-2 has also been described as negative prognostic factor for HCC progression [58]. PAI-1 has also been demonstrated to play an important role in fibrotic processes. It was suggested that the pro-fibrotic effect of PAI-1 results from the ability of this protein to recruit increasing numbers of macrophages and myofibroblasts to fibrotic tissues [59]. Therefore, these two proteins may be indicators for a chronic inflammatory status in tumorassociated fibroblasts.

Apart from proteins indicating an activated inflammatory state of the tumor-associated fibroblasts, we also identified IGF-II, a known tumor promoter [60-62], in all kinds of tumor-associated fibroblasts (Figure 4). In case of tumor-associated fibroblasts of skin, lung and liver, identification of IGF-II was patient-dependent. This may reflect the fact that we are working with primary cells, which represent biological material prone to individual variability. However, in case of the liver, up-regulation of IGF-II was already observed by others [63]. In tumor-associated BM fibroblasts, IGF-II was robustly detected by us in all samples, compatible with the notion that IGF-II contributes to multiple myeloma tumor cell growth [64]. IGF-II was apparently constitutively synthesized in the tumorassociated BM fibroblasts, because the protein was detectable not only in the supernatant, but also in the cytoplasm of these cells (Figure 4). That IGF-II was detected in control and IL-1 $\beta$ treated cells as well, although in lower amounts, may reflect the fact that BM fibroblasts are closely related to BM mesenchymal stem cells, which have been described to be characterized precisely by the expression of markers such as IGF-II [27]. A possible explanation for the appearance of IGF-II in the proteome profiles of tumor-associated skin and lung fibroblasts could also be that these fibroblasts may at least partly be derived from freshly recruited BM precursor cells. Actually, it is not yet clear whether tumor-associated fibroblasts arise from local fibroblasts, from the BM or rather result from local EMT [6,15].

Interestingly, the primary lung fibroblasts, obtained from non-cancerous lung tissue areas well beside tumors, were likewise characterized by high amounts of PLOD-2 and PAI-1 (Figure 4), whereas no IGF-II was detectable in these cells. Our FACS analysis data further have revealed that $21.5 \%$ of these cells were positive for $\alpha$-SMA, which is a marker for cancer-associated fibroblasts. It could therefore be that these fibroblasts were already part of a reactive stromal microenvironment, favorable for the further progression of the cancer $[4,65]$. Thus, the data may suggest that up-regulation of PLOD-2 and PAI-1 in fibroblasts is involved in chronic inflammatory processes which are favorable for the early phase of tumor development, whereas induction of IGF-II may play an important role in promoting tumor progression.

\section{Conclusions}

Comparative analysis of primary human cells allowed us to present protein signatures apparently characteristic for fibroblasts originating in skin, lung and BM. Furthermore, we have determined inflammation-related proteome alterations in these cells. When analyzing tumor-associated fibroblasts from clinical tissue samples, substantial individual variations were apparent. However, characteristic functional aberrations were indicated by up-regulation of IGF-II, PAI-1 and PLOD-2. Monitoring such candidate markers could provide important information about the state of the tumor microenvironment of individual samples, independent of the tumor type. Using such information may support the development of improved anticancer strategies.

\section{Methods}

\section{Cell isolation and cell culture conditions}

All kinds of fibroblasts were grown in fibroblast basal medium (FBM; Lonza Clonetics, \#CC-3131) supplemented with one FGM BulletKit (Lonza Clonetics, \#CC-3130) and $10 \%$ FCS, in a humidified $37^{\circ} \mathrm{C}$-tempered atmosphere containing $5 \% \mathrm{CO}_{2}$. Normal human lung fibroblasts (NHLF) were purchased from Lonza (Lonza Clonetics, \#CC-2512). In parallel, primary human fibroblasts were isolated from lung biopsies of patients suffering from lung adenocarcinoma, either from non-cancerous, or from cancer-associated tissue areas. Each tissue sample was treated for five hours with $200 \mathrm{u} / \mathrm{ml}$ of collagenase IV (Eubio, Wothington Biochemicals, \#LS004188), and then placed into a culture flask containing FBM (including one FGM BulletKit and 10\% FCS) and incubated at $37^{\circ} \mathrm{C} / 5 \% \mathrm{CO}_{2}$ for about five days until fibroblasts grew out of the tissue. Tissue material was then removed and fibroblasts were allowed to grow until reaching $75 \%$ of confluence. All samples were obtained with written consent of the respective donor and the approval of the Ethics Committee of the Medical University of Vienna. Human skin fibroblasts were isolated from skin punches and BM fibroblasts from BM aspirates as described previously $[17,21]$. For each cell type, at least three biological replicates, corresponding to approximately $10^{6}$ cells each, were prepared and subjected to the subsequent sample processing steps. Fibroblasts obtained from lung biopsies were characterized by FACS analysis prior to further processing.

\section{FACS analysis}

FACS analysis was performed as previously described [17]. Shortly, primary human lung fibroblasts were collected when they had reached approximately $75 \%$ of confluence. $5 \times 10^{4}-5 \times 10^{5}$ cells were resuspended in $50 \mu \mathrm{l}$ 
PBS and unspecific binding was blocked using Beriglobin (CSL Behring; diluted 1:8 in PBS/BSA/Azid). $10 \mu$ directly fluorochrome-conjugated antibody was added and after washing and fixing the cells, they were subjected to flow cytometric analysis using a LSRII instrument (BD Biosciences) and the FlowJo software (TreeStar). Following murine monoclonal antibodies (mABs) were used: FITCconjugated mAbs specific for alpha-smooth muscle actin (Sigma, \#F3777, 1:50), CD45 (Acris, \#SM3025F, 1:5), CD31 (Acris, \#BM4047F, 1:5) and CD34 (BD Pharmingen, \#555821, 1:5); phycoerythrin (PE)-conjugated mAbs specific for CD90 (Eubio, \#SM1170R, 1:3) and CD54 (BD Pharmingen, \#555511, 1:20).

\section{Inflammatory activation of fibroblasts}

For inflammatory activation, NHLF were treated for 24 hours with IL-1 $\beta$. Cells were cultivated in FBM (including one FGM BulletKit and 10\% FCS), supplemented with $10 \mathrm{ng} / \mathrm{ml}$ IL-1 $\beta$ (Sigma-Aldrich, \#I9401). After that, cells were further cultivated for 24 hours, now in FCS-free EBM-2 medium, as described in Cell fractionation, in order to obtain serum-free cell supernatants. Skin- and BM-derived primary fibroblasts, raised as previously described, $[17,21]$ were treated with IL- $1 \beta$ in the same way.

\section{Cell fractionation}

Cell fractionation was performed as previously described [17]. In short, cells were washed with EBM-2 (Lonza) without FCS and then cultivated for 24 hours in this serum-free medium to obtain the fraction of secreted proteins without contamination of highabundant serum proteins coming from FCS. Supernatants were sterile filtered $(0.2 \mu \mathrm{m}$, FP POINT $2-\mathrm{S}$, Schleicher \& Schuell, Whatman) and ethanol precipitated. For the isolation of cytoplasmic proteins cells were lysed in lysis buffer $(10 \mathrm{mM} \mathrm{HEPES} / \mathrm{NaOH}$, $\mathrm{pH} 7.4,0.25 \mathrm{M}$ sucrose, $10 \mathrm{mM} \mathrm{NaCl}, 3.5 \mathrm{mM} \mathrm{MgCl}_{2}$, 0.5\% Triton X-100, $1 \mathrm{mM}$ EGTA; protease inhibitors) and fibroblasts were pressed 12 times through a $23 \mathrm{~g}$ syringe to induce cell lysis [66]. The cytoplasmic proteins were separated from nuclei by down-centrifuging the nuclei for 5 minutes at $2300 \mathrm{~g}$ and were subsequently ethanol precipitated. To obtain the nuclear extract, the remaining pellet was lysed with $100 \mathrm{mM}$ Tris/ $\mathrm{HCl} \mathrm{pH}$ 7.4, $1 \mathrm{mM}$ EDTA pH 7.5, $500 \mathrm{mM} \mathrm{NaCl}$ and afterwards diluted in $10 \mathrm{mM}$ Tris/ $\mathrm{HCl} \mathrm{pH} \mathrm{7.4,}$ $1 \mathrm{mM}$ EDTA pH7.5, 0.5\% NP-40 (including protease inhibitors). After centrifugation at $2300 \mathrm{~g}$ for 5 minutes, the proteins in the resulting supernatant were ethanol precipitated. Afterwards, proteins of all fractions were pelletized by centrifugation for 20 minutes at $4750 \mathrm{~g}$ at $4^{\circ} \mathrm{C}$ and dissolved in sample buffer (7.5 M urea, 1.5 M thiourea, 4\% CHAPS, 0.05\% SDS, $100 \mathrm{mM}$ DDT).

\section{SDS-PAGE for subsequent shotgun analysis}

$50 \mu \mathrm{g}$ proteins of each fraction were loaded on a $12 \%$ polyacrylamid gel. Electrophoresis was performed until complete separation of a pre-stained molecular marker (Dual Color, Biorad, Hercules, CA). Proteins in the gels were fixed with $50 \%$ methanol/10\% acetic acid for $30 \mathrm{mi}-$ nutes and silver stained as described [67]. Lanes were then cut into 6 to 8 slices of different molecular weights, and proteins digested with trypsin as described below.

\section{Digestion with trypsin}

The digestion with trypsin was performed as described before [68]. In brief, proteins in gel slices were destained, reduced with DTT and alkylated with iodacetamide before they were digested with trypsin (sequencing grade, Roche) overnight at $37^{\circ} \mathrm{C}$. After elution, the peptides were forwarded to LC-MS/MS analysis.

\section{Mass spectrometry}

Mass spectrometry was performed as described previously [66]. In short, peptides were separated by nanoflow LC using the HPLC-Chip technology from Agilent, equipped with a $40 \mathrm{nl}$ Zorbax 300SB-C18 trapping column and a $75 \mu \mathrm{m}$ x $150 \mathrm{~mm}$ Zorbax 300SB-C18 separation column. For peptide elution a gradient from $0.2 \%$ formic acid and $2 \%$ ACN to $0.2 \%$ formic acid and $40 \%$ ACN over 60-80 minutes was applied. Peptide identification was accomplished by MS/MS analysis with an iontrap mass spectrometer (XCT-Ultra, Agilent) equipped with an orthogonal nanospray ion source. The MS/MS data were interpreted by the Spectrum Mill MS Proteomics Workbench software (Version A.03.03, Agilent) searching against the SwissProt/UniProtKB protein database for human proteins (Version 12/2010 containing 20328 entries). Peptides were included in the result files when their SpectrumMill score was above 13. Peptides scoring between 9 and 13 were also included if precursor $\mathrm{m} / \mathrm{z}$ value, retention time and MS2 pattern matched to a reference spectrum scoring above 13. This corresponded to a false discovery rate of less than $1 \%$ as described previously [17]. Furthermore, only proteins which were identified in at least one of the cell types with at least two distinct peptides were included in the result files. Concerning protein inference, the smallest number of proteins necessary to explain all observed peptides as described for ProteinProphet [69] was chosen.

\section{Data interpretation}

Data interpretation was supported by the Griss proteomics database engine (GPDE) [70,71]. The GPDE software can be downloaded freely from http://www.ebi.ac.uk/ pride/legacy/. A semi-quantitative assessment of protein abundance was achieved by using the number of distinct peptides identified per protein, as described previously 
[72]. Alternatively, a semi-quantitative assessment was achieved by determination of the average "emPAI" (exponentially modified protein abundance index) value of an identified protein, according to Ishihama et al. [73]. Limits for peptide identification were thereby set from $500 \mathrm{Da}$ to 4500 Da. Furthermore, in order to visualize the abundances of a protein in the different fibroblasts, the emPAI values were transformed into red tones, increased color intensities corresponding to increased emPAI values. We used colored cell symbols for each selected protein; cytoplasm, nucleus and supernatant were colored according to the emPAI values determined for the respective fractions (see Figures 1 and 4). Successful inflammatory activation of fibroblasts was demonstrated by the up-regulation of several proteins known to be induced during an inflammatory response (Table 1). Significance of the up-regulation was determined using chi-squared tests for IL-6, GROalpha and CXCL5 in IL-1 $\beta$ treated skin fibroblasts, as well as for one protein (IL-8) in IL-1 $\beta$ treated skin, lung and BM fibroblasts (Figure 3). The significance level therefor was set to $95 \%$.

\section{Additional files}

Additional file 1: Figure S1. FACS analysis of primary lung fibroblasts obtained from non-cancerous and cancerous tissue areas. Cells were characterized by FACS analysis, which showed that cells were positive for fibroblast-specific markers CD90, but negative for leukocyte, endothelial cell and hematopoietic stem cell markers CD45, CD31 and CD34 respectively. All samples contained cells which were inflammatory activated, as demonstrated by positive CD54-staining. A certain amount of the cells showed also a positive staining for a-SMA, characterizing a myofibroblast phenotype of cancer-associated fibroblasts.

Additional file 2: Table S1. Proteome profile of NHLF. Accession, Swiss-Prot accession numbers; name, protein names; peptides, the number of distinct peptides identified for each protein.

Additional file 3: Table S2. Proteome profile of primary lung fibroblasts. Accession, Swiss-Prot accession numbers; name, protein names; peptides, the number of distinct peptides identified for each protein.

Additional file 4: Table S3. Proteome profile of skin fibroblasts. Accession, Swiss-Prot accession numbers; name, protein names; peptides, the number of distinct peptides identified for each protein.

Additional file 5: Table S4. Proteome profile of BM fibroblasts. Accession, Swiss-Prot accession numbers; name, protein names; peptides, the number of distinct peptides identified for each protein.

Additional file 6: Table S5. Proteome profile of NHLF treated with IL-1 $\beta$. Accession, Swiss-Prot accession numbers; name, protein names; peptides, the number of distinct peptides identified for each protein.

Additional file 7: Table S6. Proteome profile of skin fibroblasts treated with IL-1 $\beta$. Accession, Swiss-Prot accession numbers; name, protein names; peptides, the number of distinct peptides identified for each protein.

Additional file 8: Table S7. Proteome profile of BM fibroblasts treated with IL-1 $\beta$. Accession, Swiss-Prot accession numbers; name, protein names; peptides, the number of distinct peptides identified for each protein.

Additional file 9: Table S9. Proteome profile of melanoma-associated fibroblasts. Accession, Swiss-Prot accession numbers; name, protein names; peptides, the number of distinct peptides identified for each protein.

Additional file 10: Table S10. Proteome profile of multiple myeloma-associated fibroblasts. Accession, Swiss-Prot accession numbers; name, protein names; peptides, the number of distinct peptides identified for each protein.

Additional file 11: Table S11. Proteome profile of HCC-associated fibroblasts. Accession, Swiss-Prot accession numbers; name, protein names; peptides, the number of distinct peptides identified for each protein

Additional file 12: Table S8. Proteome profile of lung carcinoma-associated fibroblasts. Accession, Swiss-Prot accession numbers; name, protein names; peptides, the number of distinct peptides identified for each protein.

Additional file 13: Table S12. emPAI values for all proteins listed in Figure 2. For each protein the emPAl values determined by us in the different sub-cellular fractions (sn, cell supernatant; cyt, cytoplasmic fraction; nuc, nuclear fraction) of the respective cell type and cell state are indicated. AccNr, Swiss-Prot accession number.

Additional file 14: Table S13. emPAl values for all proteins listed in Table 1. For each protein the emPAI values determined by us in the different sub-cellular fractions (sn, cell supernatant; cyt, cytoplasmic fraction; nuc, nuclear fraction) of the respective cell type and cell state are indicated. AccNr, Swiss-Prot accession number.

\section{Abbreviations}

BM: Bone marrow; ECM: Extracellular matrix; EMT: Epithelial-mesenchymal transition; FBM: Fibroblast basal medium; FCS: Fetal calf serum; FITC: Fluorescein isothiocyanate; GPDE: Griss proteomics database engine; HCC: Hepatocellular carcinoma; IGF-II: Insulin-like growth factor II;

IL-1ß: Interleukin-1beta; LC-MS/MS: Liquid chromatography-tandem mass spectrometry; MMP: Matrix metalloproteinase; NHLF: Normal human lung fibroblasts; PAI-1: Plasminogen activator inhibitor 1; PDGFR- $\beta$ : beta-type platelet-derived growth factor; PLOD-2: Procollagen-lysine,2-oxoglutarate 5-dioxygenase 2; PRIDE: Proteomics identification database (http://www.ebi. ac.uk/pride).

\section{Competing interests}

The authors declare that that have no competing interests.

\section{Authors' contributions}

AS performed the majority of the experiments, especially cell isolation, cell fractionation and all parts of proteome profiling, analyzed the data, prepared the figures, and wrote the manuscript; $A M$ and $A B$ supported AS with cell fractionation and proteolytic digests and $A M$ was responsible for FACS analyses; HJA and VP were responsible for the organization of patient materials; CG contributed to the overall research strategy, performed mass spectrometry analyses and contributed to data analyses. All authors read and approved the final manuscript.

\section{Acknowledgments}

This study was supported by a grant of the FWF Austrian Science Fund (grant number L670-B13), The funder had no role in study design, data collection and analysis, decision to publish, or preparation of the manuscript. We would like to thank Dr. Verena Haudek-Prinz for practical support and Editha Bayer, Thomas Mohr and Rosa-Maria Weiss for excellent technical assistance.

\section{Author details}

${ }^{1}$ Faculty of Chemistry, Department of Analytical Chemistry, University of Vienna, Vienna, Austria. ${ }^{2}$ Department of Medicine I, Institute of Cancer Research, Medical University of Vienna, Austria; Christian Doppler Laboratory for the Diagnosis and Regeneration of Cardiac and Thoracic Diseases, Medical University Vienna, Vienna, Austria. ${ }^{3}$ Department of Dermatology, Medical University of Vienna, Vienna, Austria. ${ }^{4}$ Department of Thoracic Surgery, Division of Surgery, Medical University Vienna, Vienna, Austria; Christian Doppler Laboratory for the Diagnosis and Regeneration of Cardiac and Thoracic Diseases, Medical University Vienna, Vienna, Austria.

Received: 1 September 2014 Accepted: 17 October 2014

Published: 21 November 2014 


\section{References}

1. Flavell SJ, Hou TZ, Lax S, Filer AD, Salmon M, Buckley CD: Fibroblasts as novel therapeutic targets in chronic inflammation. Br J Pharmacol 2008, 153(Suppl 1):S241-S246.

2. Buckley CD, Pilling D, Lord JM, Akbar AN, Scheel-Toellner D, Salmon M: Fibroblasts regulate the switch from acute resolving to chronic persistent inflammation. Trends Immunol 2001, 22:199-204

3. Naylor AJ, Filer A, Buckley CD: The role of stromal cells in the persistence of chronic inflammation. Clin Exp Immunol 2013, 171:30-35.

4. Kalluri R, Zeisberg M: Fibroblasts in cancer. Nat Rev Can 2006 6:392-401.

5. Hinz B, Phan SH, Thannickal VJ, Prunotto M, Desmouliere A, Varga J, De Wever O, Mareel M, Gabbiani G: Recent developments in myofibroblast biology: paradigms for connective tissue remodeling. Am J Pathol 2012 180:1340-1355.

6. Marsh T, Pietras K, MCAllister SS: Fibroblasts as architects of cancer pathogenesis. Biochim Biophys Acta 1832, 2013:1070-1078.

7. Bissell MJ, Hines WC: Why don't we get more cancer? A proposed role of the microenvironment in restraining cancer progression. Nat Med 2011, 17:320-329.

8. Hanahan D, Weinberg RA: Hallmarks of cancer: the next generation. Cell 2011, 144:646-674

9. De Wever O, Demetter $\mathrm{P}$, Mareel M, Bracke M: Stromal myofibroblasts are drivers of invasive cancer growth. Int J Cancer 2008, 123:2229-2238.

10. Hadler-Olsen E, Winberg JO, Uhlin-Hansen L: Matrix metalloproteinases in cancer: their value as diagnostic and prognostic markers and therapeutic targets. Tumour Biol 2013, 34:2041-2051.

11. Sugimoto $H$, Mundel TM, Kieran MW, Kalluri R: Identification of fibroblast heterogeneity in the tumor microenvironment. Cancer Biol Ther 2006, 5:1640-1646.

12. Pilling D, Fan T, Huang D, Kaul B, Gomer RH: Identification of markers that distinguish monocyte-derived fibrocytes from monocytes, macrophages, and fibroblasts. PloS one 2009, 4:e7475

13. Rinn JL, Bondre C, Gladstone HB, Brown PO, Chang HY: Anatomic demarcation by positional variation in fibroblast gene expression programs. PLoS Genetics 2006, 2:e119.

14. Chang HY, Chi JT, Dudoit S, Bondre C, van de Rijn M, Botstein D, Brown PO: Diversity, topographic differentiation, and positional memory in human fibroblasts. Proc Natl Acad Sci U S A 2002, 99:12877-12882.

15. Postlethwaite $A E$, Shigemitsu $H$, Kanangat $S$ : Cellular origins of fibroblasts: possible implications for organ fibrosis in systemic sclerosis. Curr Opin Rheumatol 2004, 16:733-738.

16. Paulitschke V, Gruber S, Hofstatter E, Haudek-Prinz V, Klepeisz P, Schicher N, Jonak C, Petzelbauer P, Pehamberger H, Gerner C, Kunstfeld R: Proteome analysis identified the PPARgamma ligand 15d-PGJ2 as a novel drug inhibiting melanoma progression and interfering with tumor-stroma interaction. PloS one 2012, 7:e46103.

17. Slany A, Haudek-Prinz V, Meshcheryakova A, Bileck A, Lamm W, Zielinski C, Gerner C, Drach J: Extracellular Matrix Remodeling by Bone Marrow Fibroblast-like Cells Correlates with Disease Progression in Multiple Myeloma. J Proteome Res 2014, 13:844-854.

18. Slany A, Haudek-Prinz V, Zwickl H, Stattner S, Grasl-Kraupp B, Gerner C: Myofibroblasts are important contributors to human hepatocellular carcinoma: Evidence for tumor promotion by proteome profiling. Electrophoresis 2013, 34:3315-3325.

19. Rothlein R, Mainolfi EA, Kishimoto TK: Treatment of inflammation with anti-ICAM-1. Res Immunol 1993, 144:735-739. discussion 754-762.

20. De Boeck A, Hendrix A, Maynard D, Van Bockstal M, Daniels A, Pauwels P Gespach C, Bracke M, De Wever O: Differential secretome analysis of cancer-associated fibroblasts and bone marrow-derived precursors to identify microenvironmental regulators of colon cancer progression. Proteomics 2013, 13:379-388.

21. Slany A, Paulitschke V, Haudek-Prinz V, Meshcheryakova A, Gerner C: Determination of cell type-specific proteome signatures of primary human leukocytes, endothelial cells, keratinocytes, hepatocytes, fibroblasts and melanocytes by comparative proteome profiling. Electrophoresis 2014, 35:1428-1438.

22. Salza R, Peysselon F, Chautard E, Faye C, Moschcovich L, Weiss T, PerrinCocon L, Lotteau V, Kessler E, Ricard-Blum S: Extended interaction network of procollagen C-proteinase enhancer-1 in the extracellular matrix. Biochem J 2014, 457:137-149.
23. Kato A, Okamoto O, Ishikawa K, Sumiyoshi H, Matsuo N, Yoshioka H, Nomizu M, Shimada T, Fujiwara S: Dermatopontin interacts with fibronectin, promotes fibronectin fibril formation, and enhances cell adhesion. $J$ Biol Chem 2011, 286:14861-14869.

24. Denk AE, Braig S, Schubert T, Bosserhoff AK: Slit3 inhibits activator protein 1-mediated migration of malignant melanoma cells. Int J Mol Med 2011, 28:721-726

25. Bedekovics J, Kiss A, Beke L, Karolyi K, Mehes G: Platelet derived growth factor receptor-beta (PDGFRbeta) expression is limited to activated stromal cells in the bone marrow and shows a strong correlation with the grade of myelofibrosis. Virchows Arch 2013, 463:57-65.

26. Donovan J, Shiwen X, Norman J, Abraham D: Platelet-derived growth factor alpha and beta receptors have overlapping functional activities towards fibroblasts. Fibrogenesis Tissue Repair 2013, 6:10.

27. Halfon S, Abramov N, Grinblat B, Ginis I: Markers distinguishing mesenchymal stem cells from fibroblasts are downregulated with passaging. Stem Cells Dev 2011, 20:53-66.

28. Hida Y, Hamada J: Differential expressions of matrix metalloproteinases, a disintegrin and metalloproteinases, and a disintegrin and metalloproteinases with thrombospondin motifs and their endogenous inhibitors among histologic subtypes of lung cancers. Anticancer Agents Med Chem 2012, 12:744-752.

29. Denkers IA, Beelen RH, Ossenkoppele GJ, De Jong-de Boer AJ, Langenhuijsen MM: Differences of cellular composition and adhesion molecule expression in "leukemic" as compared with "normal" human long-term bone marrow cultures. Ann Hematol 1992, 64:210-216.

30. Novince CM, Koh AJ, Michalski MN, Marchesan JT, Wang J, Jung Y, Berry JE, Eber MR, Rosol TJ, Taichman RS, McCauley LK: Proteoglycan 4, a novel immunomodulatory factor, regulates parathyroid hormone actions on hematopoietic cells. Am J Pathol 2011, 179:2431-2442.

31. Novince CM, Michalski MN, Koh AJ, Sinder BP, Entezami P, Eber MR, Pettway GJ, Rosol TJ, Wronski TJ, Kozloff KM, McCauley LK: Proteoglycan 4: a dynamic regulator of skeletogenesis and parathyroid hormone skeletal anabolism. J Bone Miner Res 2012, 27:11-25.

32. Bae S, Park CW, Son HK, Ju HK, Paik D, Jeon CJ, Koh GY, Kim J, Kim H: Fibroblast activation protein alpha identifies mesenchymal stromal cells from human bone marrow. Br J Haematol 2008, 142:827-830.

33. Hu Q, Guo C, Li Y, Aronow BJ, Zhang J: LMO7 mediates cell-specific activation of the Rho-myocardin-related transcription factor-serum response factor pathway and plays an important role in breast cancer cell migration. Mol Cell Biol 2011, 31:3223-3240.

34. Nakatani K, Okuyama H, Shimahara Y, Saeki S, Kim DH, Nakajima Y, Seki S, Kawada N, Yoshizato K: Cytoglobin/STAP, its unique localization in splanchnic fibroblast-like cells and function in organ fibrogenesis. Lab Invest 2004, 84:91-101.

35. Yan C, Ding X, Wu L, Yu M, Qu P, Du H: Stat3 downstream gene product chitinase 3-like 1 is a potential biomarker of inflammation-induced lung cancer in multiple mouse lung tumor models and humans. PloS one 2013, 8:e61984.

36. Bell SM, Schreiner CM, Wert SE, Mucenski ML, Scott WJ, Whitsett JA: $\mathrm{R}$-spondin 2 is required for normal laryngeal-tracheal, lung and limb morphogenesis. Development 2008, 135:1049-1058.

37. Foronjy R, Imai K, Shiomi T, Mercer B, Sklepkiewicz P, Thankachen J, Bodine $P, D^{\prime}$ Armiento J: The divergent roles of secreted frizzled related protein-1 (SFRP1) in lung morphogenesis and emphysema. Am J Pathol 2010, 177:598-607.

38. Groffen AJ, Buskens CA, Van Kuppevelt TH, Veerkamp JH, Monnens LA, van den Heuvel LP: Primary structure and high expression of human agrin in basement membranes of adult lung and kidney. Eur J Biochem 1998, 254:123-128.

39. Nguyen G, Delarue F, Burckle C, Bouzhir L, Giller T, Sraer JD: Pivotal role of the renin/prorenin receptor in angiotensin II production and cellular responses to renin. J Clin Invest 2002, 109:1417-1427.

40. Gallagher PE, Cook K, Soto-Pantoja D, Menon J, Tallant EA: Angiotensin peptides and lung cancer. Curr Cancer Drug Targets 2011, 11:394-404.

41. Liao W, Bao Z, Cheng C, Mok YK, Wong WS: Dendritic cell-derived interferon-gamma-induced protein mediates tumor necrosis factor-alpha stimulation of human lung fibroblasts. Proteomics 2008, 8:2640-2650.

42. Brinckmann J, Kim S, Wu J, Reinhardt DP, Batmunkh C, Metzen E, Notbohm $H$, Bank RA, Krieg T, Hunzelmann N: Interleukin 4 and prolonged hypoxia induce a higher gene expression of lysyl hydroxylase 2 and an altered 
cross-link pattern: important pathogenetic steps in early and late stage of systemic scleroderma? Matrix Biol 2005, 24:459-468.

43. Wu J, Reinhardt DP, Batmunkh C, Lindenmaier W, Far RK, Notbohm H, Hunzelmann N, Brinckmann J: Functional diversity of lysyl hydroxylase 2 in collagen synthesis of human dermal fibroblasts. Exp Cell Res 2006, 312:3485-3494.

44. Bock O, Hoftmann J, Theophile K, Hussein K, Wiese B, Schlue J, Kreipe H: Bone morphogenetic proteins are overexpressed in the bone marrow of primary myelofibrosis and are apparently induced by fibrogenic cytokines. Am J Pathol 2008, 172:951-960.

45. Brentnall TA: Arousal of cancer-associated stromal fibroblasts: palladinactivated fibroblasts promote tumor invasion. Cell Adh Migr 2012, 6:488-494.

46. Ronty MJ, Leivonen SK, Hinz B, Rachlin A, Otey CA, Kahari VM, Carpen OM: Isoform-specific regulation of the actin-organizing protein palladin during TGF-beta1-induced myofibroblast differentiation. J Invest Dermatol 2006, 126:2387-2396.

47. Xin H, Pereira-Smith OM, Choubey D: Role of IFI 16 in cellular senescence of human fibroblasts. Oncogene 2004, 23:6209-6217.

48. Li T, Diner BA, Chen J, Cristea IM: Acetylation modulates cellular distribution and DNA sensing ability of interferon-inducible protein IFI16. Proc Natl Acad Sci U S A 2012, 109:10558-10563.

49. Sponza S, De Andrea M, Mondini M, Gugliesi F, Gariglio M, Landolfo S: Role of the interferon-inducible IFI16 gene in the induction of ICAM-1 by TNF-alpha. Cell Immunol 2009, 257:55-60.

50. Anisowicz A, Messineo M, Lee SW, Sager R: An NF-kappa B-like transcription factor mediates IL-1/TNF-alpha induction of gro in human fibroblasts. J Immunol 1991, 147:520-527.

51. Brach MA, Henschler R, Mertelsmann RH, Herrmann F: Regulation of M-CSF expression by M-CSF: role of protein kinase $C$ and transcription factor NF kappa B. Pathobiology 1991, 59:284-288.

52. van de Stolpe A, Caldenhoven E, Stade BG, Koenderman L, Raaijmakers JA, Johnson JP, van der Saag PT: 12-O-tetradecanoylphorbol-13-acetate- and tumor necrosis factor alpha-mediated induction of intercellular adhesion molecule- 1 is inhibited by dexamethasone. Functional analysis of the human intercellular adhesion molecular-1 promoter. J Biol Chem 1994, 269:6185-6192.

53. Basile A, Sica A, D'Aniello E, Breviario F, Garrido G, Castellano M, Mantovani A, Introna M: Characterization of the promoter for the human long pentraxin PTX3. Role of NF-kappaB in tumor necrosis factor-alpha and interleukin-1 beta regulation. J Biol Chem 1997, 272:8172-8178.

54. Bocian C, Urbanowitz AK, Owens RT, lozzo RV, Gotte M, Seidler DG: Decorin potentiates interferon-gamma activity in a model of allergic inflammation. J Biol Chem 2013, 288:12699-12711.

55. Hayashi N, Yoshimoto T, Izuhara K, Matsui K, Tanaka T, Nakanishi K T helper 1 cells stimulated with ovalbumin and IL-18 induce airway hyperresponsiveness and lung fibrosis by IFN-gamma and IL-13 production. Proc Natl Acad Sci U S A 2007, 104:14765-14770.

56. Nikolaou K, Sarris M, Talianidis I: Molecular pathways: the complex roles of inflammation pathways in the development and treatment of liver cancer. Clin Cancer Res 2013, 19:2810-2816.

57. Bozoky B, Savchenko A, Csermely P, Korcsmaros T, Dul Z, Ponten F, Szekely L, Klein G: Novel signatures of cancer-associated fibroblasts. Int J Cancer 2013, 133:286-293

58. Noda T, Yamamoto H, Takemasa I, Yamada D, Uemura M, Wada H, Kobayashi S, Marubashi S, Eguchi H, Tanemura M, Umeshita K, Doki Y, Mori $M$, Nagano H: PLOD2 induced under hypoxia is a novel prognostic factor for hepatocellular carcinoma after curative resection. Liver Int 2012, 32:110-118.

59. Norman J: Fibrosis and progression of autosomal dominant polycystic kidney disease (ADPKD). Biochim Biophys Acta 2011, 1812:1327-1336.

60. Maloney EK, McLaughlin JL, Dagdigian NE, Garrett LM, Connors KM, Zhou XM, Blattler WA, Chittenden T, Singh R: An anti-insulin-like growth factor I receptor antibody that is a potent inhibitor of cancer cell proliferation. Cancer Res 2003, 63:5073-5083.

61. Bergman D, Halje M, Nordin M, Engstrom W: Insulin-like growth factor 2 in development and disease: a mini-review. Gerontology 2013, 59:240-249.

62. Greenall SA, Bentley JD, Pearce LA, Scoble JA, Sparrow LG, Bartone NA, Xiao $X$, Baxter RC, Cosgrove LJ, Adams TE: Biochemical characterization of individual human glycosylated pro-insulin-like growth factor (IGF)-II and big-IGF-II isoforms associated with cancer. J Biol Chem 2013, 288:59-68.
63. Breuhahn K, Schirmacher P: Reactivation of the insulin-like growth factor-II signaling pathway in human hepatocellular carcinoma. World J Gastroenterol 2008, 14:1690-1698.

64. Jelinek DF, Witzig TE, Arendt BK: A role for insulin-like growth factor in the regulation of IL-6-responsive human myeloma cell line growth. J Immunol 1997, 159:487-496.

65. Barron DA, Rowley DR: The reactive stroma microenvironment and prostate cancer progression. Endocr Relat Cancer 2012, 19:R187-R204.

66. Gundacker NC, Haudek VJ, Wimmer H, Slany A, Griss J, Bochkov V, Zielinski C, Wagner O, Stockl J, Gerner C: Cytoplasmic proteome and secretome profiles of differently stimulated human dendritic cells. J Proteome Res 2009, 8:2799-2811.

67. Mortz E, Krogh TN, Vorum H, Gorg A: Improved silver staining protocols for high sensitivity protein identification using matrix-assisted laser desorption/ionization-time of flight analysis. Proteomics 2001, 1:1359-1363.

68. Slany A, Haudek VJ, Gundacker NC, Griss J, Mohr T, Wimmer H, Eisenbauer M, Elbling L, Gerner C: Introducing a new parameter for quality control of proteome profiles: consideration of commonly expressed proteins. Electrophoresis 2009, 30:1306-1328.

69. Nesvizhskii Al, Keller A, Kolker E, Aebersold R: A statistical model for identifying proteins by tandem mass spectrometry. Anal Chem 2003, 75:4646-4658.

70. Griss J, Gerner C: GPDE: a biological view on PRIDE. J Proteomics Bioinform 2009, 2:167-174.

71. Griss J, Haudek-Prinz V, Gerner C: GPDE: A biological proteomic database for biomarker discovery and evaluation. Proteomics 2011, 11:1000-1004.

72. Wimmer H, Gundacker NC, Griss J, Haudek VJ, Stattner S, Mohr T, Zwickl H, Paulitschke V, Baron DM, Trittner W, Kubicek M, Bayer E, Slany A, Gerner C: Introducing the CPL/MUW proteome database: interpretation of human liver and liver cancer proteome profiles by referring to isolated primary cells. Electrophoresis 2009, 30:2076-2089.

73. Ishihama Y, Oda Y, Tabata T, Sato T, Nagasu T, Rappsilber J, Mann M: Exponentially modified protein abundance index (emPAl) for estimation of absolute protein amount in proteomics by the number of sequenced peptides per protein. Mol Cell Proteomics 2005, 4:1265-1272.

doi:10.1186/1559-0275-11-4

Cite this article as: Slany et al:: Plasticity of fibroblasts demonstrated by tissue-specific and function-related proteome profiling. Clinical Proteomics 2014 11:41.

\section{Submit your next manuscript to BioMed Central and take full advantage of:}

- Convenient online submission

- Thorough peer review

- No space constraints or color figure charges

- Immediate publication on acceptance

- Inclusion in PubMed, CAS, Scopus and Google Scholar

- Research which is freely available for redistribution 